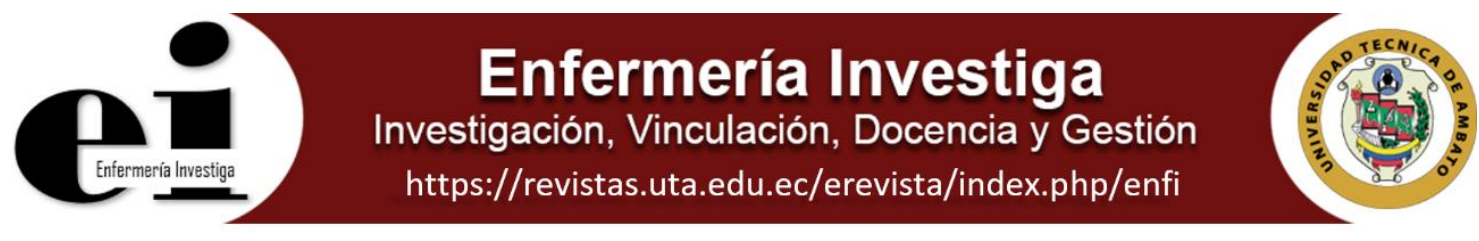

\title{
LA SALUD MENTAL DEL PERSONAL SANITARIO ANTE LA PANDEMIA DEL COVID-19
}

\section{THE MENTAL HEALTH OF HEALTHCARE PERSONNEL IN THE COVID-19 PANDEMIC}

Indira López Izurieta¹ https://orcid.org/0000-0002-8907-9856, Ignacio López Izurieta² https://orcid.org/0000-0002-9635-189.

\begin{abstract}
${ }^{1}$ Docente de la Carrera de Enfermería, Universidad Central del Ecuador, Quito, Ecuador. Estudiante del Programa de Doctorado en EnfermeríaUniversidad de Concepción, Chile

${ }^{2}$ Universidad Central del Ecuador, Quito, Ecuador. Estudiante del Master en Prevención de Riesgos Laborales-Universidad Internacional de la Rioja, España.
\end{abstract}

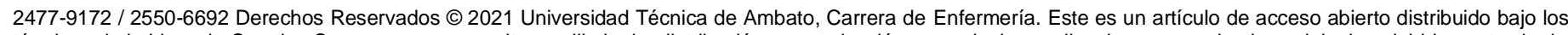

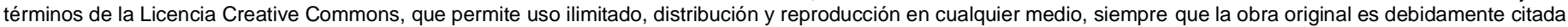

Recibido: 01 de octubre 2020

Aceptado: 01 de diciembre 2020

\section{Resumen}

A finales del año 2019, se reportaron los primeros casos de COVID-19 en la ciudad de Wuhan-China, que inicialmente se consideró como una neumonía de origen desconocido y posteriormente se convirtió en una gran amenaza para los sistemas sanitarios, que afecta al personal de salud por la repercusión emocional que implica enfrentarse a situaciones complejas y estresantes, que alteran los mecanismos de regulación emocional y la capacidad de adaptación a la vida cotidiana, por la excesiva carga laboral y disminución de los periodos de descaso, produciendo mayor predisposición a presentar ansiedad, depresión, estrés, insomnio y fatiga, que limitarían las funciones del personal de salud, afectando de forma directa la contención de la pandemia del COVID-19

En el equipo de salud el personal de enfermería tiene mayor vulnerabilidad a factores de riesgo biológicos y sociales para desarrollar afecciones de la salud mental como la ansiedad, por su alta exposición al virus al realizar su trabajo y el temor al contagio tanto personal como familiar.

A pesar de todos los recursos empleados para contrarrestar la propagación del COVID-19, actualmente el personal de salud necesita estrategias globales de autocuidado y apoyo psicológico, para disminuir los efectos negativos en la salud mental, como técnicas cognitivo-conductuales para detener los ciclos negativos de pensamientos, controlar los factores estresantes, gestionar adecuadamente el tiempo, técnicas de autoayuda con terapia cognitivo-conductual en línea, terapias de conversación, grupos de apoyo o psicoterapia e intervenciones organizacionales.

Palabras claves: infección por coronavirus; salud mental; pandemia; ansiedad.

\begin{abstract}
At the end of 2019, the first cases of COVID-19 were reported in the city of Wuhan-China, which was initially considered as pneumonia of unknown origin and later became a great threat to health systems, affecting staff of health due to the emotional repercussion that implies facing complex and stressful situations, which alter the mechanisms of emotional regulation and the ability to adapt to daily life, due to the excessive workload and reduction of periods of rest, producing a greater predisposition to present anxiety, depression, stress, insomnia and fatigue, which would limit the functions of health personnel, directly affecting the containment of the COVID-19 pandemic

In the health team, the nursing staff has greater vulnerability to biological and social risk factors for developing mental health conditions such as anxiety, due to their high exposure to the virus when carrying out their work and the fear of both personal and family contagion.

Despite all the resources used to counteract the spread of COVID-19, health personnel currently need comprehensive strategies for self-care and psychological support, to reduce the negative effects on mental health, such as cognitive-behavioral techniques to stop negative cycles thinking, managing stressors, managing time appropriately, self-help techniques with online cognitive-behavioral therapy, talk therapy, support groups or psychotherapy, and organizational interventions.
\end{abstract}

Keywords: coronavirus infection; mental health; pandemic; anxiety. 


\section{INTRODUCCIÓN}

A finales del año 2019, en el mes de diciembre se reportaron los primeros casos de la enfermedad COVID-19 en la ciudad China de Wuhan, el brote inicial del Coronavirus fue en un mercado de dicha ciudad, diagnosticados en ese momento como casos de neumonía de origen desconocido (1-6). El agente etiológico es el Betacoronavirus de ARN denominado por el Comité Internacional de Taxonomía de los Virus como el coronavirus de tipo 2 causante del síndrome respiratorio agudo severo (SRASCoV-2) (1-5), para el mes de enero de 2020 la Organización Mundial de la Salud (OMS) declaró en Emergencia Sanitaria a la comunidad internacional, a causa del COVID-19, por los acelerados niveles de expansión y complejidad, que en el mes de marzo paso de epidemia a pandemia de forma oficial (4). El contagio de la COVID-19 se da de forma directa, a través de pequeñas gotas de 5 micras expulsadas durante episodios de tos o estornudos, que ingresan por medio de la mucosa respiratorias y/o conjuntivales, fómites como madera, acero, plástico que son llevadas de manera accidental por el huésped hasta sus mucosas (7).

Los pacientes con COVID-19 se clasifican según la gravedad en (7):

- Leve: se caracteriza presentar síntomas en las vías respiratorias altas, fiebre $\mathrm{y} / \mathrm{o}$ mialgias, con una evolución no complicada.

- Moderada: confirmada por medio de radiografía de tórax como una neumonía leve, con saturación de oxígeno al aire ambiente $\geq 90 \%$ y no requiere asistencia respiratoria (7).

- Grave. presencia de insuficiencia respiratoria aguda, con infiltrados pulmonares en la radiografía de tórax que requiere ingreso en la Unidad de Cuidados Intensivos (UCI).

La Pandemia COVID-19 representa una gran amenaza a los sistemas de salud, sobretodo de los países con recursos económicos medios y bajos, ya que para afrontar esta crisis sanitaria se necesita un gran apoyo económico y de recursos humanos, por lo tanto es fundamental la implementación de medidas profilácticas, para no saturar los sistemas sanitarios como: el cierre de lugares de aglomeración de personas, medidas de aislamiento social y el fomento del distanciamiento social, pero que reducen fuertemente a la economía y la productividad de un gran número de países (5,8-10), esta pandemia ha afectado a todos los sectores de la sociedad, en particular a las organizaciones y como consecuencia a sus trabajadores, por los diversos cambios en el ámbito laboral como: disminución de las nóminas, amplios horarios de trabajo y masificación de los centros de salud y hospitales (1,9-13), por lo que es necesario fomentar la salud mental para mantener el bienestar de los trabajadores, especialmente los profesionales de salud: médicos, enfermeras, auxiliares, entre otros; ya que se encuentran expuestos de forma directa al COVID-19 $(3,14)$, por tal motivo, actualmente se ha convertido en un punto prioritario la salud mental de los trabajadores sanitario (4), porque han generado síntomas depresivos, alteraciones del sueño, ansiedad, temor, estrés e ira (15), por lo tanto, es fundamental la protección de la salud mental ya que las alteraciones psicológicas inciden en la atención, comprensión y toma de decisiones, que a su vez afecta de forma directa la contención de la pandemia(16), por estas razones el objetivo de la revisión es analizar y comparar los estudios realizados en torno a la actual pandemia del COVID-19 y su posible incidencia en la salud mental del personal de salud de primera línea, que está enfrentando la emergencia sanitaria

\section{AFECTACIÓN EN LA SALUD MENTAL A CAUSA DE LA PANDEMIA DEL COVID-19 EN EL PERSONAL DE SALUD:}

Según Huang y col., del 7 al 14 de febrero del 2020, se realizaron las primeras investigaciones en el mundo con el objetivo de conocer el estado de salud mental del equipo sanitario y establecer las bases fundamentales para la intervención psicológica (17), en esta investigación realizada en China se aplicó la Escala de autoevaluación para la ansiedad (SAS) y la Escala de autoevaluación para el Trastorno de estrés Postraumático (PTSD-SS), estableciendo que el personal de enfermería presentó mayor ansiedad que el personal médico, por su alta exposición al virus en momento de realizar su trabajo, con un mayor número de casos en el sexo femenino, por el temor al contagio, tanto personal como familiar y son más vulnerables a los factores de riesgo biológicos y sociales para desarrollar alguna afección de salud mental, en los médicos a mayor formación académica se incrementa el nivel de ansiedad (1518), por estas razones se debe crear e implementar equipos de intervención psicológica y proporcionar la atención y el asesoramiento necesario al personal sanitario $(15,17)$.

En otro estudio realizado en Latinoamérica por Samaniego y col., sobre la afectación a la salud mental del personal de hospitales en Paraguay destinados para atención exclusiva de pacientes con COVID-19, establecieron que el personal sanitario está sometido a factores que contribuyen al desarrollo de alteraciones en la salud mental como: turnos extendidos de trabajo, excesiva carga laboral y disminución de los periodos de descaso (19), donde los profesionales de enfermería del sexo femenino tiene una mayor predisposición a presentar ansiedad, depresión, estrés, insomnio y fatiga, relacionado con la naturaleza de su trabajo que conlleva a mayor exposición y permanencia con los pacientes (20-22).

Ecuador presenta 198.244 casos confirmados por COVID-19 hasta el 7 de diciembre del 2020 (23), lo que aún sigue complicado en estos meses la situación sanitaria del país, por lo que el Ministerio de Salud Pública del Ecuador (MSP), implemento medidas para mejorar el déficit que existe en insumos y equipos médicos, incrementado la disponibilidad de los equipos de protección personal (EPP) para los trabajadores sanitarios y el número de camas del sistema salud nacional, para de esta forma aliviar la carga de pacientes (24-25), restando importancia a la salud mental del personal sanitario que está afrontando la emergencia sanitaria.

\section{RECOMENDACIONES PARA FOMENTAR LA SALUD MENTAL DEL PERSONAL DE SALUD DURANTE LA PANDEMIA COVID-19}

A pesar de todos los recursos empleados para contrarrestar la propagación del COVID-19, actualmente el personal de salud necesita estrategias globales de autocuidado para disminuir los efectos negativos en la salud mental, que se debe considerar una parte importante del bienestar y la salud en general. La Organización Panamericana de la Salud/Organización Mundial de la Salud estableció las siguientes recomendaciones para fortalecer la salud mental del personal de salud $(18,26)$ : 
- El control del estrés es un elemento importante para mantener el bienestar holístico del equipo de salud (18,27-32).

- Apoyar las necesidades diarias básicas de los profesionales sanitarios fomentando un estilo de vida saludable y el autocuidado, con una alimentación balanceada con requerimientos adecuados de antioxidantes como frutas y hortalizas, descansar y hacer pausas durante el trabajo o entre turnos, hacer ejercicio domiciliario a pesar del distanciamiento social, evitar el exceso de redes sociales y la cobertura mediática de COVID-19, incluir el uso de técnicas como la relajación muscular progresiva o la meditación, para evitar pensamientos negativos, además, evitar el consumo de sustancias como el alcohol, tabaco y demás drogas, ya que pueden perjudicar el bienestar físico y mental (18).

- Fortalecer y mantener la resiliencia personal permite al trabajador de salud gestionar sus experiencias negativas y la discriminación por su entorno (al considerarlos como un riesgo) $(18,28)$.

- Brindar apoyo psicológico utilizando técnicas cognitivoconductuales para detener los ciclos negativos de pensamientos, controlar los factores estresantes, gestionar adecuadamente el tiempo, con técnicas de manejo de autoayuda con Terapia cognitivo-conductual (TCC) en línea, terapias de conversación, grupos de apoyo 0 psicoterapia e intervenciones organizacionales. $(18,28,30)$.

- Adoptar una actitud positiva de solución de problemas y participar en iniciativas solidarias para propiciar emociones agradables

- Aplicar pruebas de tamizaje para detectar manifestaciones de psicopatología que pudieran poner en riesgo a los trabajadores de la salud $(31,32)$

- Las personas con problemas de salud mental preexistentes deben continuar con el tratamiento y estar atentas a la aparición de síntomas nuevos o al agravamiento de sus síntomas $(31,32)$.

\section{CONCLUSIONES}

El avance de la COVID-19 ha sido devastador para el mundo en especial en Latinoamérica, dejando además a la vista pública las desigualdades estructurales en los sistemas de salud, la inequidad, la falta de fortalecimiento en la atención primaria en salud, la necesidad de inversión en la provisión de equipos de protección para el personal de salud

En la actualidad la pandemia de COVID-19, presenta un momento crítico que genera en el personal de salud ansiedad, depresión, estrés, insomnio y fatiga, por lo que se debe implementar equipos de intervención psicológica y proporcionar la atención y el asesoramiento necesario al personal sanitario, con estrategias globales de autocuidado, para disminuir los efectos negativos en la salud mental, como técnicas cognitivoconductuales para detener los ciclos negativos de pensamientos, controlar los factores estresantes, gestionar adecuadamente el tiempo, técnicas de autoayuda con terapia cognitivo-conductual en línea, terapias de conversación, grupos de apoyo o psicoterapia e intervenciones organizacionales.

\section{REFERENCIAS}

1. Trilla A. One world, one health: The novel coronavirus COVID-19 epidemic. Medicina clínica. 2020;154(5):175-177. Disponible en: https://www.ncbi.nIm.nih.gov/pmc/articles/PMC7094554/

2. Fernández González L, Bravo Valenzuela P. Expertos y redes sociales: ¿Cómo comunicarnos en tiempos de pandemia?. Rev. Méd. Chile. 2020; 148(4):560-561. Disponible en: https://scielo.conicyt.cl/scielo.php?script=sci_arttext\&pid=S003498872020000400560\&lng=es.http://dx.doi.org/10.4067/s0034-98872020000400560

3. Vera-Villarroel P. Psicología y covid-19: un análisis desde los procesos psicológicos básicos. Panamerican Journal of Neuropsychology. 2020; 14(1):10-18. Disponible en: http://www.cnps.cl/index.php/cnps/article/viewFile/393/440

4. Cruz A, Gutiérrez A, Zaldívar E. Gestión de seguridad psicológica del personal sanitario en situaciones de emergencia por COVID-19 en el contexto hospitalario o de aislamiento. Rev Cubana Enferm. 2020;36(2). Disponible en: http://www.revenfermeria.sld.cu/index.php/enf/article/view/3704

5. Alarcón-Guzmán R. Perspectivas éticas en el manejo de la pandemia COVID-19 y de su impacto en la salud mental. Rev Neuropsiquiatr. 2020;83(2):97-103. Disponible en: http://www.scielo.org.pe/scielo.php?script=sci_arttext\&pid=S0034$85972020000200097 \&$ Ing=es. http://dx.doi.org/10.20453/rnp.v83i2.3757.

6. Huarcaya-Victoria J. Consideraciones sobre la salud mental en la pandemia de COVID-19.Rev Peruana de medicina $\begin{array}{lllll}\text { experimental y salud } & \text { pública. }\end{array}$ http://www.scielo.org.pe/scielo.php?pid=S172646342020000200327\&script=sci_arttext

7. Huerta Sáenz I, Elías Estrada J, Campos Del Castillo K, Muñoz Taya R, Coronado J. Características materno perinatales de gestantes COVID-19 en un hospital nacional de Lima, Perú. Rev. Perú. ginecol. obstet. 2020;66(2):00003. Disponible en: http://www.scielo.org.pe/scielo.php?script=sci_arttext\&pid=S2304-51322020000200003\&lng=es. http://dx.doi.org/10.31403/rpgo.v66i2245.

8. Juárez-García A. Síndrome de burnout en personal de salud durante la pandemia COVID-19: un semáforo naranja en la salud mental. Rev Salud UIS. 2020;52(4). Disponible en: https://revistas.uis.edu.co/index.php/revistasaluduis/article/view/11528

9. Organización Mundial de la Salud. Salud mental 2020. Ginebra: OMS; 2020. Disponible en: https://www.who.int/topics/mental_health/es/\#: :text=Salud\%20mental,ausencia\%20de\%20afecciones\%200\%20enfermed ades\%C2\%BB.

10. Wu Z, Mc Googan JM. Characteristics of and Important Lessons From the Coronavirus Disease 2019 (COVID-19) Outbreak in China: Summary of a Report of 72314 Cases From the Chinese Center for Disease Control and Prevention. JAMA. 
2020;323(13):1239. Disponible en: https://doi.org/10.1001/jama.2020.2648

11. Peraza de Aparicio C. Salud laboral frente a la pandemia del COVID-19 en Ecuador. Medisur. 2020;18(3): 507-511. Disponible en: http://scielo.sld.cu/scielo.php?script=sci_arttext\&pid=S1727-897X2020000300507\&lng=es. Epub 02-Jun2020.

12. Torre-Muñoz V., Farias-Cortés J.D., Reyes-Vallejo L.A., Guillen-Díaz-Barriga C. Riesgos y daños en la salud mental del personal sanitario por la atención a pacientes con Covid-19. Rev. Mex. Urol. 2020;80(3):1-9. Disponible en: https://www.medigraphic.com/pdfs/uro/ur-2020/ur203i.pdf

13. World Health Organization. COVID-19 situation reports. 2020.Disponible en:https://www.who.int/emergencies/diseases/novelcoronavirus2019/situation-reports

14. Benavides F. La salud de los trabajadores y la COVID-19. Archivos de Prevención de Riesgos Laborales.2020;14(661):1988. Disponible en: https://archivosdeprevencion.eu/index.php/apr//article/view/50/21

15. Lozano-Vargas A. Impacto de la epidemia del Coronavirus (COVID-19) en la salud mental del personal de salud y en la población general de China. Rev Neuropsiquiatr.2020; 83(1):51-56. Disponible en: http://www.scielo.org.pe/scielo.php?script=sci_arttext\&pid=S0034-85972020000100051\&lng=es. http://dx.doi.org/10.20453/rnp.v83i1.3687

16. Kang L, Li Y, Hu S, et al. The mental health of medical workers in Wuhan, China dealing with the 2019 novel coronavirus. Lancet Psychiatry. 2020; 7(3):14. Disponible en: https://www.ncbi.nlm.nih.gov/pmc/articles/PMC7129673/

17. Huang JZ, Han MF, Luo TD, Ren AK, Zhou XP. [Mental health survey of medical staff in a tertiary infectious disease hospital for COVID-19]. Chinese Journal of Industrial Hygiene and Occupational Diseases,Chinese. 2020;38(3):192-195. Disponible en: 10.3760/cma.j.cn121094-20200219-00063. PMID: 32131151

18. Organización mundial de la salud. Consideraciones psicosociales y de salud mental durante el brote de COVID-19. Ginebra:OMS.2020. Disponible en:

https://iris.paho.org/bitstream/handle/10665.2/52571/OPSWNMHMHCovid-1920040_spa.pdf?sequence=3\&isAllowed=y

19. Samaniego A, Urzúa A, Buenahora M, Vera-Villarroel P. Sintomatología asociada a trastornos de salud mental en trabajadores sanitarios en paraguay: efecto covid-19.Revista Interamericana de Psicología. 2020;54(1):1298. Disponible en: https://journal.sipsych.org/index.php/IJP/article/view/1298/1013

20. Muñoz Zambrano C, Rumie Díaz H, Torres Gómez G, Villarroel Julio K. Impacto en la salud mental de la(del) enfermera(o) que otorga cuidados en situaciones estresantes. Cienc. enferm. 2015; 21(1): 45-53. Disponible en: https://scielo.conicyt.cl/scielo.php?script=sci_arttext\&pid=S0717-

95532015000100005\&lng=es.http://dx.doi.org/10.4067/S0717-95532015000100005.

21. Arratia F A. Investigación y documentación histórica en enfermería. Texto \& Contexto Enfermagem.2005;14(4):567574.Disponible en: https://www.redalyc.org/articulo.oa?id=71414414

22. Zarate Grajales R. La Gestión del Cuidado de Enfermería. Index Enferm. 2004;13(44-45): 42-46. Disponible en: http://scielo.isciii.es/scielo.php?script=sci_arttext\&pid=S1132-12962004000100009\&lng=es.

23. COVID-19 EC. Las cifras del COVID-19 en Ecuador.2020. Disponible en: https://coronavirusecuador.com/

24. Comité de Operaciones de Emergencia Nacional. Informe de Situación COVID-19 Ecuador. 2020. Disponible en: https://www.gestionderiesgos.gob.ec/wp-content/uploads/2020/04/Informe-de-Situaci\%C3\%B3n-No042-CasosCoronavirus-Ecuador-29042020.pdf

25. Li Q, Guan X, Wu P, Wang X, Zhou L, Tong Y, et al. Early Transmission Dynamics in Wuhan, China, of Novel CoronavirusInfected Pneumonia. N Engl J Med. 2020;382(13):1199-1207. Disponible en: 10.1056/NEJMoa2001316. Epub 2020 Jan 29. PMID: 31995857; PMCID: PMC7121484.

26. Ferro R. Salud mental y poder. Un abordaje estratégico de las acciones en salud mental en la comunidad. Revista de Salud Pública. 2010; 14(2):47-62. Disponible en: https://revistas.unc.edu.ar/index.php/RSD/article/view/7082

27. Organización Panamericana de la Salud .Salud Mental y COVID- 19. 2020 .Disponible en: https://www.paho.org/es/saludmental-covid-19

28. Ramírez-Ortiz J, Castro-Quintero D, Lerma-Córdoba C, Yela-Ceballos F, Escobar-Córdoba F. Mental health consequences of the COVID-19 pandemic associated with social isolation. Colombian Journal of Anesthesiology. 2020;48(4):e930. Disponible en: http://www.scielo.org.co/pdf/rca/v48n4/es_2256-2087-rca-48-04-e301.pdf

29. Hernández Rodríguez J. Impacto de la COVID-19 sobre la salud mental de las personas. Medicentro Electrónica. 2020;24(3): 578-594.Disponible en: http://scielo.sld.cu/scielo.php?script=sci_arttext\&pid=S1029-30432020000300578\&lng=es. Epub 01-Jul-2020.

30. Asociación de Salud Mental y Psiquiatría Comunitaria. Guía de apoyo psicosocial durante esta epidemia de coronavirus OME-AEN; 2020. Disponible en: https://ome-aen.org/guia-de-apoyo-psicosocial-durante-esta-epidemia-de-coronavirus/

31. Muñoz-Fernández SI, Molina-Valdespino D, Ochoa-Palacios R, et al. Estrés, respuestas emocionales, factores de riesgo, psicopatología y manejo de los trabajadores de la salud durante la pandemia (COVID-19). Acta Pediatr Mex. 2020; 41 (Suplemento: 1):127-136.Disponible en: https://www.medigraphic.com/pdfs/actpedmex/apm-2020/apms201q.pdf

32. Valero Cedeño, $\quad$ Nereida $\quad$ Josefina, Vélez Cuenca María Felicidad, Duran Mojica Ányelo Alberto, Torres Portillo Mariana. Afrontamiento del COVID-19: estrés, miedo, ansiedad y depresión. Enfermería Investiga. 2020;5(3):63-70. Disponible en: https://revistas.uta.edu.ec/erevista/index.php/enfi/article/view/913 